\title{
Anti-Proliferative Activities and Apoptosis Induction by Triterpenes Derived from Eriobotrya japonica in Human Leukemia Cell Lines
}

\author{
Takuhiro Uto ${ }^{1}$, Ayana Sakamoto ${ }^{1}$, Nguyen Huu Tung ${ }^{1}$, Tsukasa Fujiki ${ }^{2}$, Kenji Kishihara ${ }^{2}$, \\ Shigeru Oiso $^{3}$, Hiroko Kariyazono ${ }^{3}$, Osamu Morinaga ${ }^{1}$ and Yukihiro Shoyama ${ }^{1, *}$
}

1 Department of Pharmacognosy, Faculty of Pharmaceutical Sciences, Nagasaki International University, 2825-7 Huis Ten Bosch, Sasebo, Nagasaki 859-3298, Japan; E-Mails: uto@niu.ac.jp (T.U.); mabo12141030@gmail.com (A.S.); tunginpc@gmail.com (N.H.T.); morinaga@niu.ac.jp (O.M.)

2 Department of Immunology, Faculty of Pharmaceutical Sciences, Nagasaki International University, 2825-7 Huis Ten Bosch, Sasebo, Nagasaki 859-3298, Japan; E-Mails: fujiki@niu.ac.jp (T.F.); kishihar@niu.ac.jp (K.K.)

3 Department of Pharmaceutical Health Care Sciences, Faculty of Pharmaceutical Sciences, Nagasaki International University, 2825-7 Huis Ten Bosch, Sasebo, Nagasaki 859-3298, Japan; E-Mails: shige@niu.ac.jp (S.O.); karihiro@niu.ac.jp (H.K.)

* Author to whom correspondence should be addressed; E-Mail: shoyama@niu.ac.jp; Tel./Fax: +81-956-20-5622.

Received: 19 December 2012; in revised form: 15 January 2013 / Accepted: 4 February 2013 / Published: 18 February 2013

\begin{abstract}
Eriobotrya japonica leaf is a traditional herbal medicine that contains numerous triterpenes, which have various pharmacological properties. In this study, we investigated the anti-proliferative activity of four triterpenes derived from E. japonica, including corosolic acid (CA), ursolic acid (UA), maslinic acid (MA) and oleanolic acid (OA), in human leukemia cell lines. CA showed the strongest anti-proliferative activity in all of the leukemia cell lines tested, but not in normal human skin fibroblast cell lines. To determine the mechanism underlying the anti-proliferative effect of $\mathrm{CA}$, we examined the effect of $\mathrm{CA}$ on molecular events known as apoptosis induction. CA induced chromatin condensation, DNA fragmentation, sub- $\mathrm{G}_{1}$ phase DNA, activation of caspase-3, -8 and -9 and the cleavage of PARP in HL-60. CA also activated Bid and Bax, leading to the loss of mitochondrial membrane potential $\left(\Delta \psi_{\mathrm{m}}\right)$ and cytochrome $\mathrm{c}$ release into the cytosol, whereas Bcl-2 and Bcl-xL were unaffected by CA. These results suggest that CA has an anti-proliferative effect on leukemia cells via the induction of apoptosis mediated by
\end{abstract}


mitochondrial dysfunction and caspase activation. CA may be a potential chemotherapeutic agent for the treatment of human leukemia.

Keywords: corosolic acid; Eriobotrya japonica; leukemia cells; apoptosis; anti-proliferation; caspase

\section{Introduction}

Eriobotrya japonica Lindl., also known as loquat, is a fruit tree in the Rosaceae family. Its leaves are listed in the Japanese Pharmacopeia and are used widely as traditional herbal medicine for the treatment of chronic bronchitis and coughs in Japan and other Asian countries [1]. Previously, we found that a crude extract from the leaves strongly inhibited the production of prostaglandin $\mathrm{E}_{2}\left(\mathrm{PGE}_{2}\right)$ and nitric oxide (NO) in lipopolysaccharide (LPS)-treated macrophages [2]. In addition, the extract of E. japonica leaf was reported to have several anti-cancer effects. E. japonica extract showed strong cytotoxicity in cervical cancer (HeLa), lung cancer (A549) and ER-negative breast cancer (MDA-MB-231) cell lines [3]. Kim et al. have reported that E. japonica extract inhibits the adhesion, migration and invasion of human breast cancer [4]. Moreover, the cell migration and invasion of B16F10 melanoma cells were down-regulated by the extract of E. japonica leaves [5].

Phytochemical investigations of E. japonica leaves led to the isolation of various triterpenes, some of which were found to possess several pharmacological properties, including anti-inflammatory, anti-tumor, antioxidative and anti-diabetes effects [6-9]. The triterpenes-rich fraction and several isolated triterpenes from $E$. japonica leaves showed the inhibitory effect on 12-O-tetradecanoylphorbol-13-acetate (TPA)-induced inflammation and Epstein-Barr virus early antigen (EBV-EA) activation induced by TPA in mice [10]. In addition, the triterpenes from E. japonica inhibited inflammatory cytokines/mediators on human lung epithelial cells (A-549) [11] and a rat model of chronic bronchitis [12]. A recent study reported that triterpenes from E. japonica exerted anti-fibrosis effects in a rat model of bleomycin-induced pulmonary fibrosis [13]. However, the anti-proliferative activities against cancer cells by triterpenes contained in E. japonica leaves are not fully understood.

Apoptosis is a highly regulated process that involves the activation of a series of molecular events leading to cell death. Apoptosis can be initiated by two principal apoptosis pathways, i.e., the death receptor (extrinsic) pathway and the mitochondrial (intrinsic) pathway [14]. The mitochondrial pathway is mainly regulated by Bcl-2 family proteins, which are divided into the pro-apoptotic proteins (e.g., Bax and Bid) and anti-apoptotic proteins (e.g., Bcl-2 and Bcl-xL) [15].

It was reported that the major triterpenes from E. japonica leaves are ursane types, such as corosolic acid (CA) and ursolic acid (UA), and oleanane types, such as maslinic acid (MA) and oleanolic acid (OA) (Figure 1) [16,17]. These major triterpenes show anti-proliferative activities against gastric cancer cells (NCI-N87), colorectal cancer (HCT15), cervical cancer (HeLa), glioblastoma (U291, U373 and T98G) and colon cancer (HT29) cell lines [18-23]. As part of the ongoing study, our current screening of medicinal plants found promising antiproliferative effects of a methanol extract from E. japonica leaves on several human cancer cell lines. In our preliminary survey, we found that the 
extract of E. japonica leaves suppresses the cell proliferation of leukemia cell lines. In the present study, we investigated the anti-proliferative effects of the major triterpenes from E. japonica leaves, i.e., $\mathrm{CA}, \mathrm{UA}, \mathrm{MA}$ and $\mathrm{OA}$, on four human leukemia cell lines. Our data defined the structure-activity correlations of these triterpenes. Furthermore, we investigated the molecular mechanism of CA-induced apoptosis using HL-60 cell lines.

Figure 1. Chemical structures of corosolic acid (CA) and ursolic acid (UA), maslinic acid (MA) and oleanolic acid (OA).
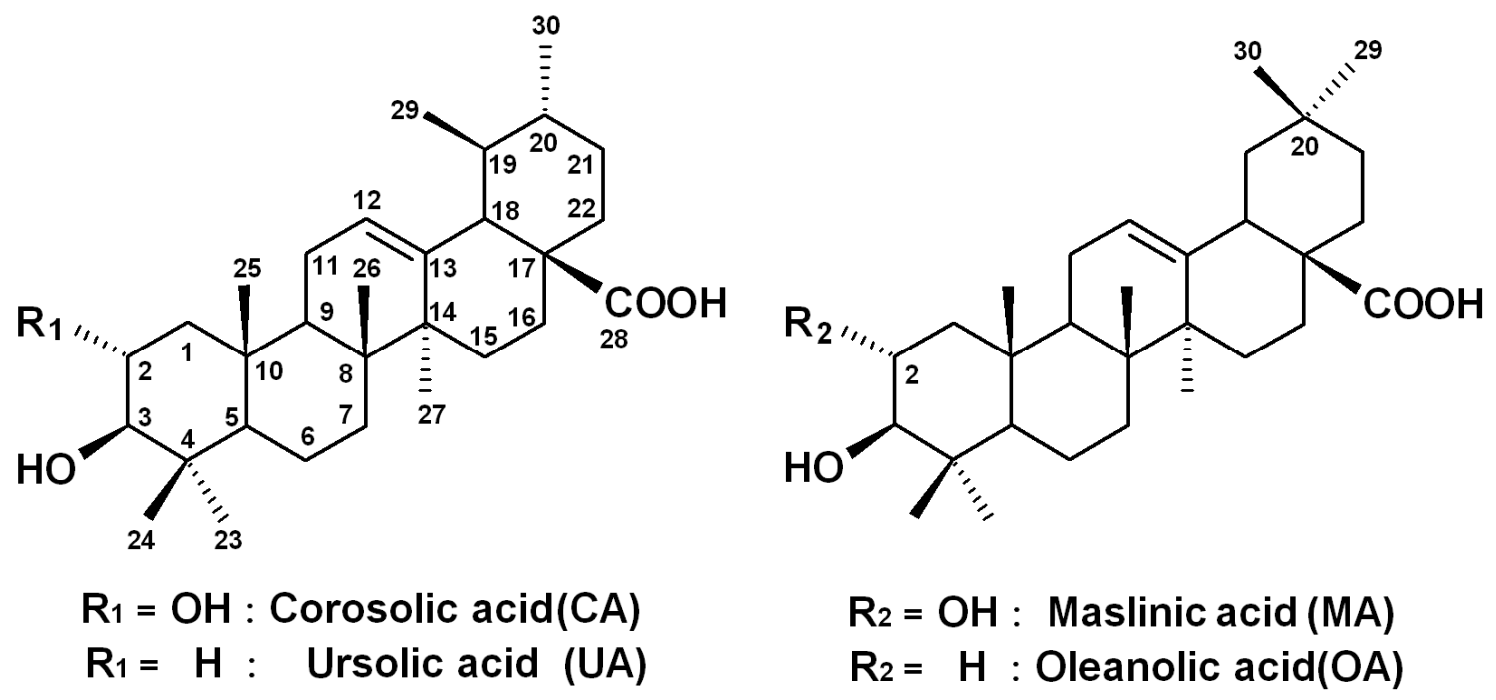

\section{Results and Discussion}

\subsection{Quality Control of E. japonica Leaves}

Because the triterpenes from E. japonica leaves might be expected to have potent bioactivities in vivo and in vitro, the quality control of these triterpenes is necessary for the constant and evident bioactivities. In our ongoing studies on the quality control of natural products, we have been investigating the preparation of monoclonal antibodies against pharmacologically active compounds and their application for quality and/or quantity analysis by using unique methods, like Eastern blotting, a high-sensitive on-membrane quantitative analysis [24-27]. Since E. japonica leaves contain various kinds of triterpenes, we performed a fingerprinting of triterpenes contained in E. japonica leaves by HPLC to confirm the importance of quality control of E. japonica. As shown in Figure 2, the HPLC fingerprint of the $\mathrm{CHCl}_{3}$ extract of E. japonica leaves showed the profile of the triterpene constituents, where four compounds were highlighted relatively as major components, i.e., CA, UA, MA and OA. The retention time of the ursan-type skeleton (CA and UA) is longer than that of the oleanane group (MA and OA). The retention time of dihydroxyl group in the A-ring (MA and CA) are shorter than that of the monohydroxyl group (OA and UA). Therefore, the quality control of E. japonica leaves for the constant bioactive evidence might be needed to confirm the quantitative determination of CA and UA. 
Figure 2. HPLC profile of four triterpenes derived from a $\mathrm{CHCl}_{3}$ extract of E. japonica leaves.

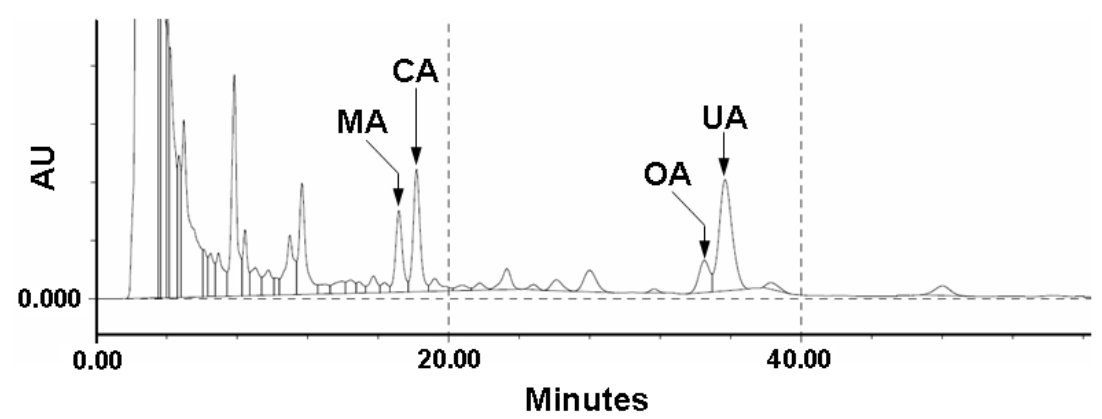

\subsection{Effects of the Four Triterpenes from E. japonica Leaves on Cell Proliferation in Human Leukemia and Normal Skin Fibroblast Cell Lines and Their Structure-Activity Correlation}

To investigate the effects of CA, UA, MA and OA on cell proliferation in leukemia cell lines (HL-60, U937, Jurkat and THP-1) and normal skin fibroblast cell lines (NHSF46 and NB1RGB), we treated the cells using $6.25,12.5$ and $25 \mu \mathrm{M}$ of each triterpene for $24 \mathrm{~h}$, followed by the MTT assay (Figure 3). CA and UA significantly suppressed cell growth in all leukemia cell lines, whereas MA and OA had weaker effects than CA and UA. The inhibitory potency against leukemia cell lines followed the order: $\mathrm{CA}>\mathrm{UA}>\mathrm{MA}=\mathrm{OA}$. However, none of the triterpenes inhibited cell proliferation in NGSF46 and NB1RGB remarkably. Thus, we suggest the following structure-activity correlations: (1) the ursane-type skeleton (CA and UA) has a greater suppressive potency than the oleanane-type skeleton (MA and $\mathrm{OA}$ ); (2) the $\mathrm{C}_{2}-\mathrm{C}_{3}$ trans-dihydroxyl group in the A-ring is important when comparing $\mathrm{CA}$ and $\mathrm{UA}$; and (3) the $\mathrm{C}_{19}-\mathrm{C}_{20}$ trans-dimethyl group in the E-ring is important when comparing $\mathrm{CA}$ and MA. The results indicated that $\mathrm{CA}$ was the most potent anti-proliferative triterpene against all of the leukemia cell lines tested, whereas it had low cytotoxicity in normal skin fibroblasts. Among four leukemia cell lines, HL-60 and U937 were more sensitive than Jurkat and THP-1 against the four triterpenes. In future studies, we will investigate the detailed mechanisms underlying the sensitivity difference among the four leukemia cell lines.

\subsection{Effect of CA on Apoptosis Induction}

We investigated whether the CA-induced anti-proliferative activity against leukemia cells was related to apoptosis induction by analyzing the characteristics of apoptosis, including nuclear morphological changes and DNA fragmentation in HL-60 and U937 cells. Both cell lines were treated with $\mathrm{CA}$ at 12.5 and $25 \mu \mathrm{M}$ for $24 \mathrm{~h}$, and the nuclear morphology of the cells was observed using Hoechst 33258 staining. As shown in Figure 4A, the control cells exhibited normal nuclear morphology, whereas the cells treated with CA displayed chromatin condensation. Furthermore, DNA fragmentation was examined based on classical DNA laddering using agarose gel electrophoresis. Figure 4B shows that the CA treatment led to the appearance of the DNA ladder in a dose- and time-dependent manner in both lines. In a parallel experiment, we also analyzed the hypodiploid DNA content (sub-G1 phase) using flow cytometry after the cellular DNA had been stained with propidium iodide (PI). CA treatment of the cells increased in the percentage of cells in the sub-G1 phase from 
$5.2 \%$ (control) to $16.4 \%(12 \mathrm{~h})$ and $61.6 \%(24 \mathrm{~h})$, gradually (Figure 4C). Overall, these results clearly indicate that CA exerted its anti-proliferative effect via the induction of apoptotic cell death.

Figure 3. Effects of the four triterpenes on cell proliferation in human leukemia and normal skin fibroblast cell lines. Cells were treated with CA, UA, MA and OA at various concentrations for $24 \mathrm{~h}$, and the cell viability was determined using the MTT assay. The data represent the mean \pm S.D. for three individual experiments. ${ }^{*} p<0.05$ compared with the control group.
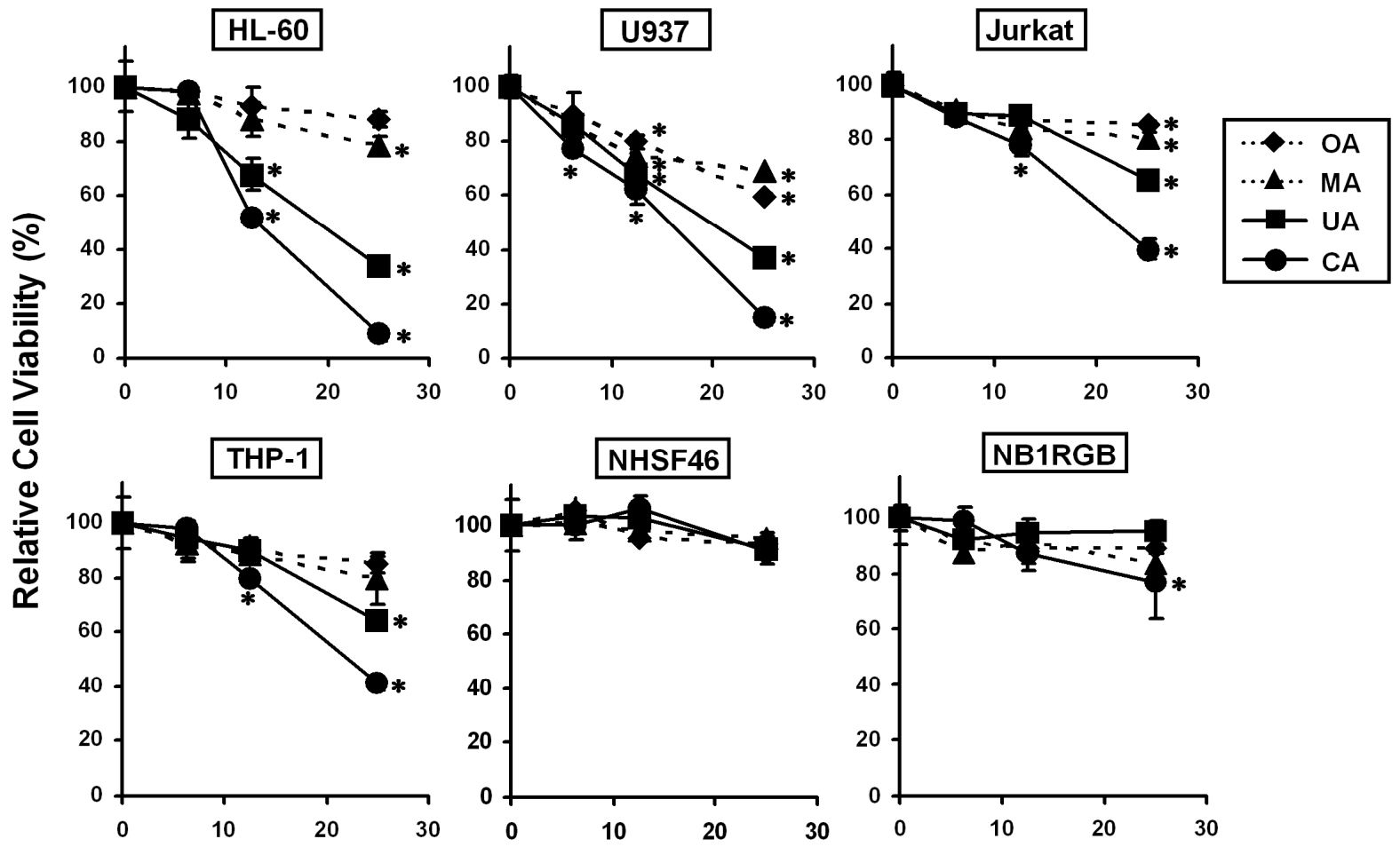

Concentration $(\mu \mathrm{M})$

\subsection{Involvement of the Caspase Cascade in CA-Induced Apoptosis}

Caspase-3 is a critical effector caspase and initiates apoptotic damage. Activation of caspase-3 requires the activation of initiator caspases, such as caspase- 8 and -9 [28]. To investigate the involvement of the caspase cascade during CA-induced apoptosis, we examined the activation of caspases in CA-treated HL-60 cells by Western blotting. As shown in Figure 5A, CA induced time-dependent activations of caspase-3, -8 and -9. Furthermore, PARP cleavage occurred in response to CA treatment. To confirm the involvement of caspases in CA-induced apoptosis, the cells were treated with $\mathrm{CA}$ in the absence or presence of caspase inhibitors, and then, the DNA fragmentation was analyzed. As shown in Figure 5B, CA-induced DNA fragmentation was abolished by pretreatment with z-VAD-FMK (broad caspase inhibitor), z-DEVF-FMK (caspase-3 inhibitor), z-IETD-FMK (caspase-8 inhibitor) and z-LEHD-FMK (caspase-9 inhibitor). These results indicate that CA-induced apoptosis involves a caspase-dependent pathway in HL-60 cells. 
Figure 4. Effect of CA on apoptosis induction. (A) Induction of chromatin condensation by CA. HL-60 and U937 cells were treated with the indicated concentration of CA for $24 \mathrm{~h}$ and stained with Hoechst 33258. The nuclear morphology was observed by fluorescent microscopy (magnification 400×); (B) Induction of DNA fragmentation by CA. HL-60 and U937 cells were treated with $\mathrm{CA}$ at various concentrations for $24 \mathrm{~h}$ or at $12.5 \mu \mathrm{M}$ for the times indicated, and the DNA fragmentation was analyzed by agarose gel electrophoresis. $\mathrm{M}$ is the 100-bp DNA marker; (C) Increase of the sub-G1 phase cells by CA. HL-60 cells were treated with $12.5 \mu \mathrm{M}$ CA for the times indicated and analyzed by flow cytometry after staining with propidium iodide (PI). The data shown are representative of three independent experiments with similar results.

A
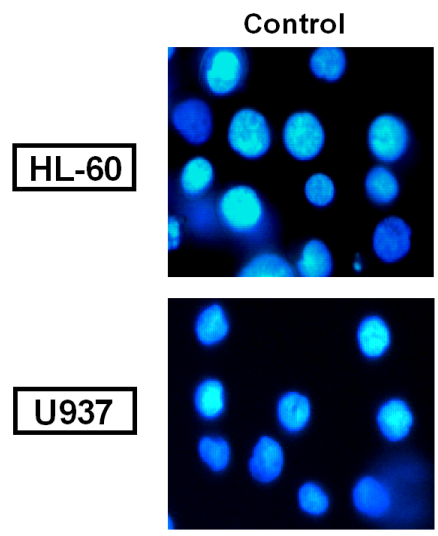

CA $12.5 \mu \mathrm{M}$
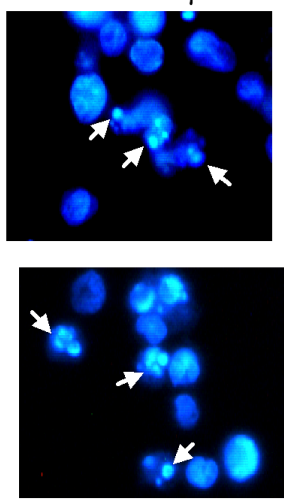

CA $25 \mu \mathrm{M}$
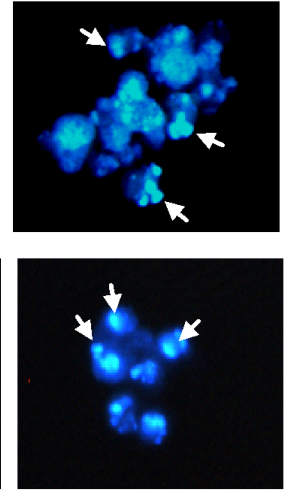

B
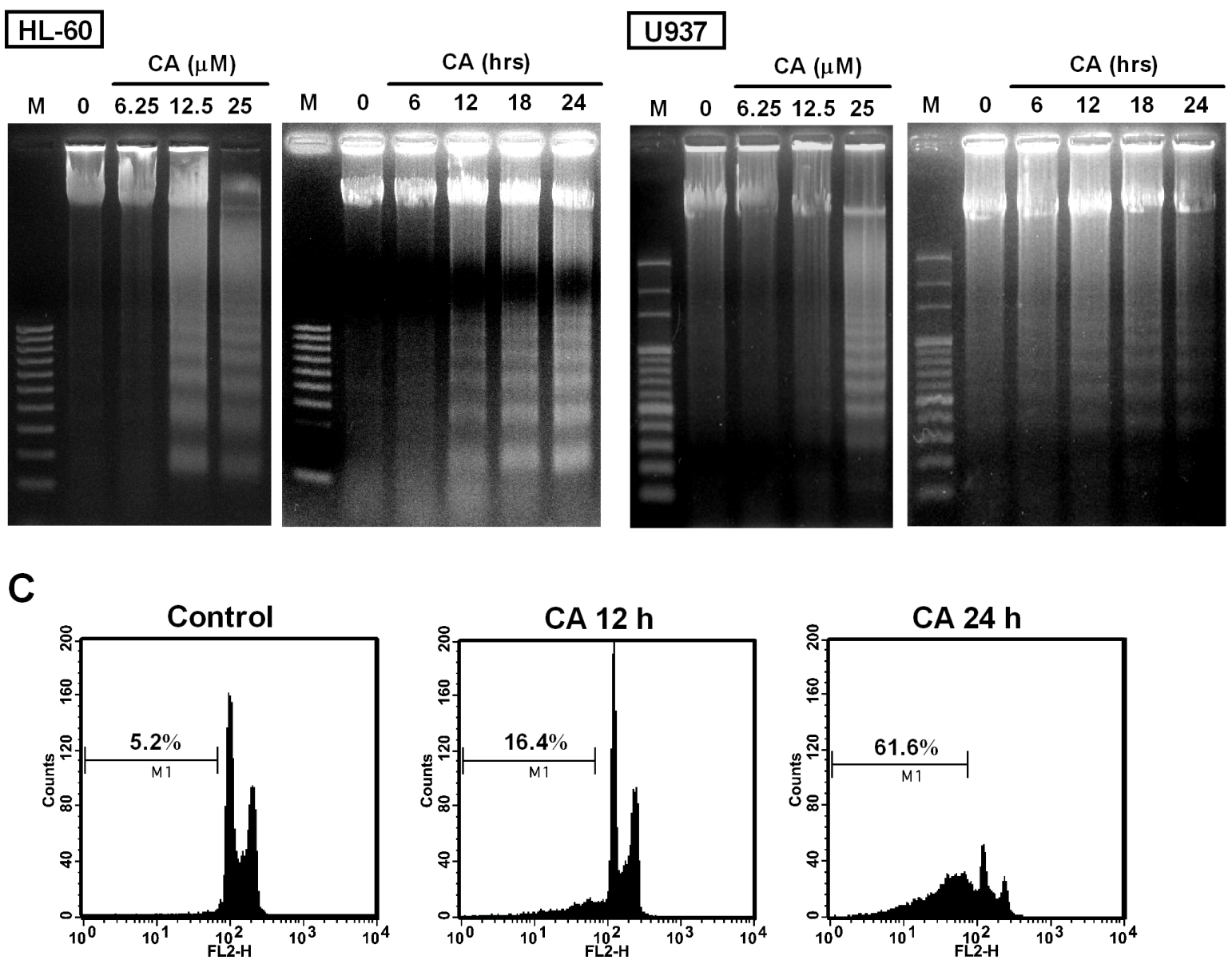
Figure 5. Involvement of the caspase cascade in CA-induced apoptosis. (A) Effect of CA on the activation of caspase-3, -8, -9 and PARP cleavage. HL-60 cells were treated with 12.5 $\mu \mathrm{M}$ CA for the times indicated. The cells were lysed, and the caspase- $3,-8,-9$, PARP and $\beta$-actin protein levels were determined by Western blotting. $\beta$-actin was used as the loading control; (B) The effect of caspase inhibitors on CA-induced DNA fragmentation. After pretreatment with $50 \mu \mathrm{M}$ caspase inhibitors for $1 \mathrm{~h}$, HL-60 cells were treated with $12.5 \mu \mathrm{M} \mathrm{CA}$ for $24 \mathrm{~h}$. DNA fragmentation was analyzed by agarose gel electrophoresis. The data shown are representative of three independent experiments with similar results.

A

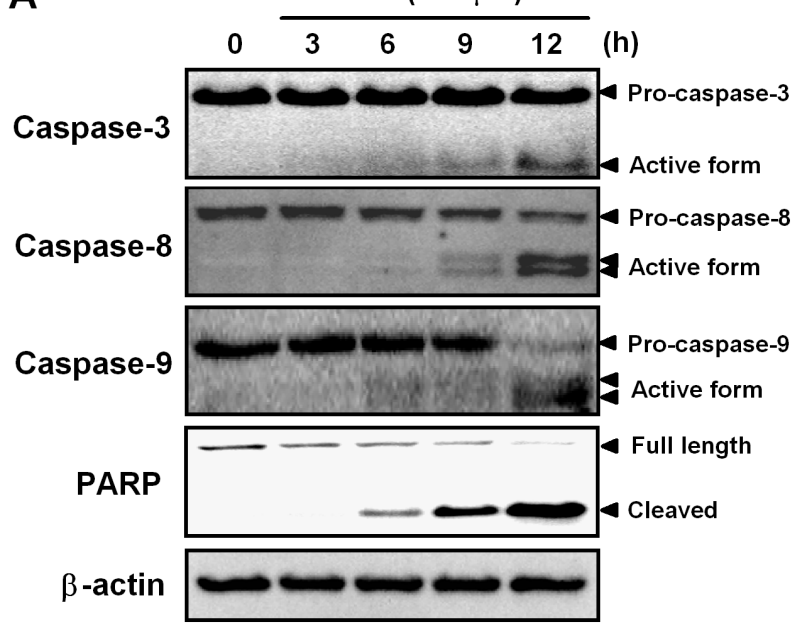

B

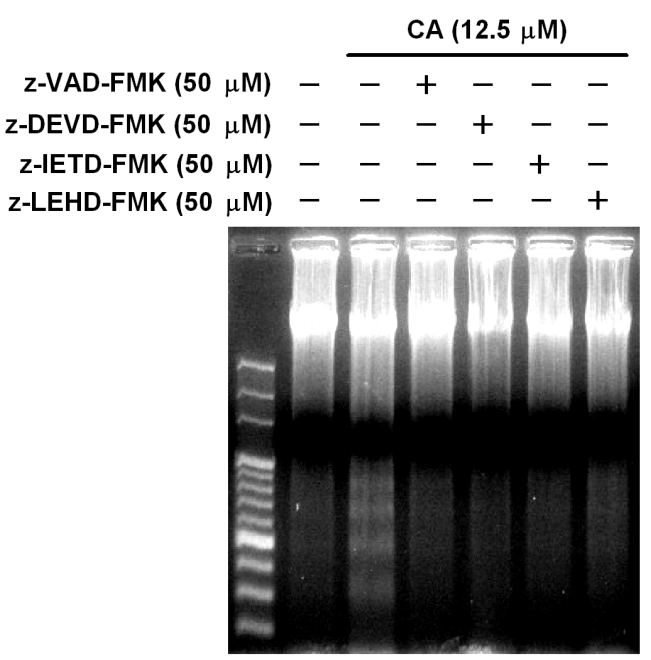

\subsection{Effect of CA on Mitochondrial Dysfunction}

Mitochondria play an essential role in apoptosis induction by a variety of death stimuli. Mitochondrial changes include the loss of the mitochondrial membrane potential $\left(\Delta \psi_{\mathrm{m}}\right)$ and cytochrome $\mathrm{c}$ release from the mitochondria to the cytosol, which subsequently leads to caspase-9-dependent activation of caspase-3 [29]. Thus, we examined the effect of CA on the $\Delta \psi_{\mathrm{m}}$ in HL-60 cells. The cells were treated with CA for $6 \mathrm{~h}$ and $12 \mathrm{~h}$, stained with JC-1, which is a sensitive mitochondrial membrane potential probe, and analyzed using flow cytometry. JC-1 can selectively enter mitochondria, depending on the membrane potential, and the JC-1 molecule spontaneously forms J-aggregates that produce intense red fluorescence. As shown in Figure 6A, the CA treatment resulted in a time-dependent decrease in the percentage of red fluorescent cells from $1.0 \%$ in the control to $12.7 \%$ and $70.7 \%$ after treatments of $6 \mathrm{~h}$ and $12 \mathrm{~h}$, respectively. Cytochrome $\mathrm{c}$ is normally located in the intermembrane space of mitochondria, and loss of the $\Delta \psi_{\mathrm{m}}$ causes the release of cytochrome $\mathrm{c}$ from mitochondria into the cytosol [30]. Western blotting showed that cytochrome c gradually accumulated in the cytosol in a time-dependent manner in response to CA treatment (Figure 6B). These results indicate that mitochondrial dysfunction is involved in CA-induced apoptosis in HL-60 cells. 
Figure 6. Effect of CA on mitochondrial dysfunction and Bcl-2 family proteins. (A) Effect of CA on the loss of $\Delta \psi_{\mathrm{m}}$. HL-60 cells were treated with $12.5 \mu \mathrm{M}$ CA for the times indicated and analyzed by flow cytometry after staining with JC-1; (B) Cytochrome c release into the cytosol after treatment with CA. HL-60 cells were treated with $12.5 \mu \mathrm{M}$ $\mathrm{CA}$ for the times indicated, and the cytosolic fraction was analyzed to detect cytochrome $\mathrm{c}$ by Western blotting. $\alpha$-Tubulin and COX-IV were used as cytosolic and mitochondrial fraction controls, respectively. The data shown are representative of three independent experiments with similar results.

A
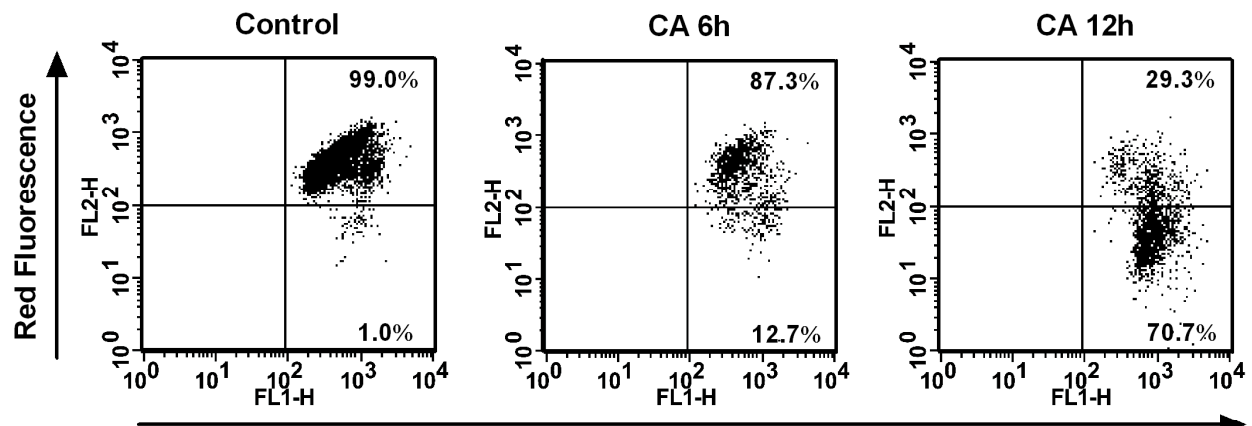

Green Fluorescence

B

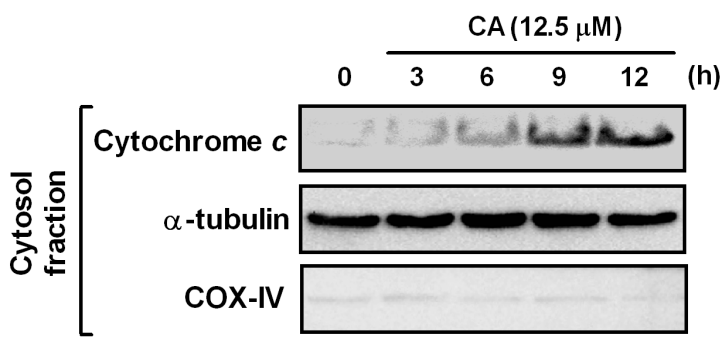

\subsection{Effect of CA on Bcl-2 Family Proteins}

Mitochondrial-mediated apoptosis regulates a balance between pro-apoptotic (e.g., Bax and Bid) and anti-apoptotic (e.g., Bcl-2 and Bcl-xL) proteins [15]. Thus, we analyzed the levels of the Bcl-2 family proteins in CA-treated HL-60 cells. Bid is a pro-apoptotic member, which is cleaved by caspase- 8 to its active form, truncated Bid (tBid). As shown in Figure 7, the CA treatment cleaved Bid protein to tBid in a time-dependent manner. By contrast, the anti-apoptotic proteins, Bcl-2 and Bcl-xL, were unaffected by $\mathrm{CA}$. Bid activation triggers the translocation of cytosolic Bax into mitochondria, which is followed by the loss of $\Delta \psi_{\mathrm{m}}$ [31]. Thus, we examined the protein level of Bax in the mitochondrial and cytosolic fractions. Our data clearly showed that CA promotes the translocation of Bax into mitochondria. Taken together, our results suggest that $\mathrm{CA}$ induces the activation of the pro-apoptotic Bid and Bax, which leads to the loss of $\Delta \psi_{\mathrm{m}}$ and the release of cytochrome c from mitochondria into the cytosol. 
Figure 7. Effect of CA on Bcl-2 family proteins. HL-60 cells were treated with $12.5 \mu \mathrm{M}$ $\mathrm{CA}$ for the times indicated. Whole cell lysates and mitochondrial and cytosolic fractions were prepared, and the levels of each protein were determined by Western blotting. $\beta$-actin, COX-IV and $\alpha$-tubulin were used as the loading controls for the whole cell lysate, mitochondrial fraction and cytosolic fraction, respectively. The data shown are representative of three independent experiments with similar results.

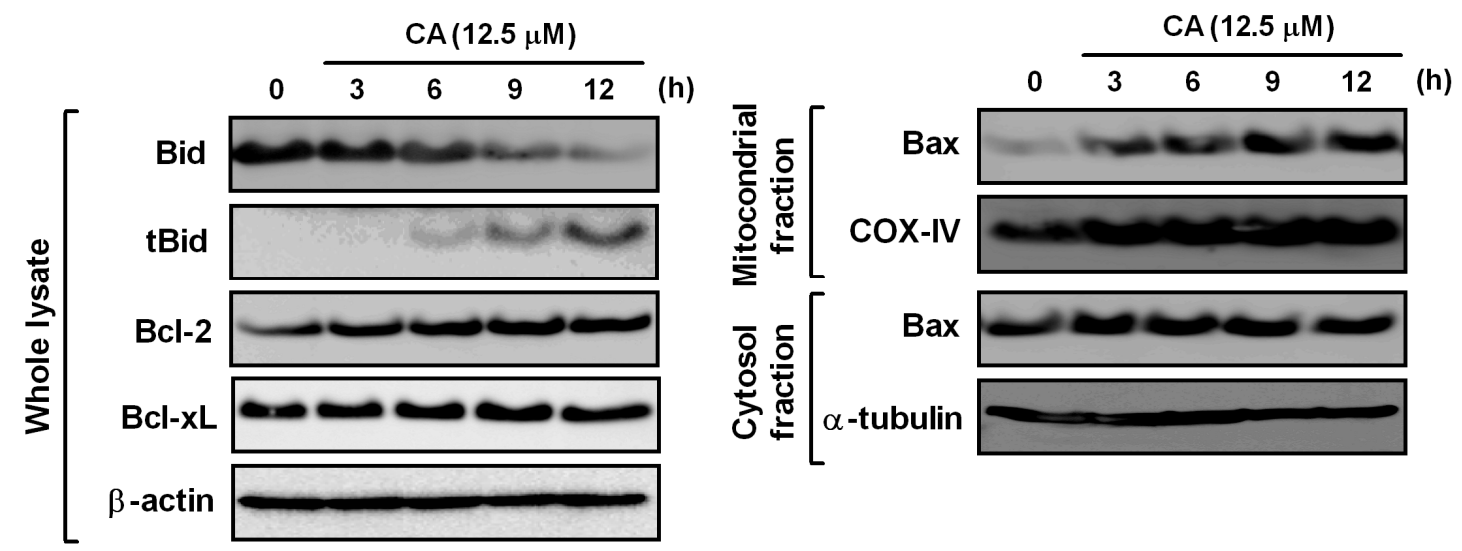

\section{Experimental Section}

\subsection{Materials}

CA and MA were purchased from Tokiwa Phytochemical (Tokyo, Japan) and Cayman Chemical (Ann Arbor, MI, USA), respectively. UA and OA were obtained from Wako Pure Chemical Industries (Osaka, Japan). E. japonica leaves were purchased from Uchida Wakan-yaku (Tokyo, Japan). Antibodies against caspase-3 (catalog number 9662), -8 (catalog number 9746), -9 (catalog number 9502), poly(ADP-ribose) polymerase (PARP), Bcl-2 family proteins, $\beta$-actin, COX-IV and $\alpha$-tubulin were obtained from Cell Signaling Technology (Beverly, MA, USA). Fetal bovine serum (FBS) was supplied by GIBCO (Gaithersburg, MD, USA). Caspase inhibitors were purchased from Calbiochem (San Diego, CA, USA). All other chemicals were obtained from Wako Pure Chemical Industries.

\subsection{HPLC Fingerprints of Triterpenes in the Crude Extract from E. japonica Leaves}

The standard triterpenes, i.e., CA, UA, MA and $\mathrm{OA}$, were dissolved in $\mathrm{MeOH}$ at a concentration of $2.0 \mathrm{mg} / \mathrm{mL}$ and stored at $4{ }^{\circ} \mathrm{C}$ until use. E. japonica leaves were extracted with $\mathrm{MeOH}$. The $\mathrm{MeOH}$ extract was suspended in water and then partitioned with $\mathrm{CHCl}_{3}$. The $\mathrm{CHCl}_{3}$ fraction $(30.0 \mathrm{mg})$ was dissolved in $\mathrm{MeOH}(1.0 \mathrm{~mL})$ and filtered with a $0.45-\mu \mathrm{m}$ syringe filter. HPLC analysis was performed using a TOSOH 8020 (Tokyo, Japan) equipped with a TSKgel ODS-100V (5 $\mu \mathrm{m}, 250 \times 4.6 \mathrm{~mm}$ ) and an UV-8020 detector (Tosoh, Tokyo, Japan). Separation was conducted using a mobile phase of methanol and $0.15 \%$ acetic acid aqueous solution $(85: 15, v / v)$. The solvent flow rate was kept constant at $1.0 \mathrm{~mL} / \mathrm{min}$ at ambient temperature throughout the analysis. 


\subsection{Purification and Isolation of Triterpenes by Preparative HPLC}

The major triterpenes from the $\mathrm{CHCl}_{3}$ fraction of $E$. japonica leaves were separated by HPLC, as described above, and were identified by the comparison with each standard compound individually. The purity of all triterpenes ( $>98 \%$ ) was confirmed by high-performance liquid chromatography.

\subsection{Cell Culture and Treatment}

HL-60 (human promyelocytic leukemia cells), U937 (human promonocytic leukemia cells), Jurkat (human acute T-cell leukemia cells), THP-1 (human monocytic leukemia cells), NHSF46 (normal human skin fibroblast cells) and NB1RGB (normal human skin fibroblast cells) were obtained from the RIKEN BioResource Center Cell Bank. HL-60, U937, Jurkat and THP-1 were maintained in RPMI1640 medium. NHSF46 and NB1RGB were grown in $\alpha$-MEM medium. All cell cultures were supplemented with $10 \% \mathrm{FBS}, 1 \%$ penicillin-streptomycin and incubated at $37{ }^{\circ} \mathrm{C}$ with $5 \% \mathrm{CO}_{2}$ in fully humidified conditions. For the cell treatments, CA, UA, MA, OA and caspase inhibitors were dissolved in DMSO and stored at $-20{ }^{\circ} \mathrm{C}$ until use. The DMSO concentrations in the cell culture medium did not exceed $0.2 \%(v / v)$, and the controls were always treated with the same amount of DMSO as that used in the corresponding experiments.

\subsection{Determination of Cell Viability}

Cell viability was determined using a 3-(4,5-dimethylthiazol-2-yl)-2,5-diphenyltetrazolium bromide (MTT) assay. Four leukemia cells $\left(1 \times 10^{4}\right.$ cells/well $)$ and fibroblast cells $\left(0.4 \times 10^{4}\right.$ cells/well $)$ were cultured in 96-well plates and treated with various concentrations of each triterpene for $24 \mathrm{~h}$. At the end of the treatment, MTT solution was added to each well, and the cells were incubated for another $4 \mathrm{~h}$. The precipitated MTT-formazan was dissolved in $0.04 \mathrm{~N} \mathrm{HCl}$-isopropanol and the amount of formazan was measured at $595 \mathrm{~nm}$ using a microplate reader (Immuno Mini NJ-2300, Nihon InterMed, Tokyo, Japan). Cell viability was expressed as a percentage relative to the control culture.

\subsection{Nuclear Staining with Hoechst 33258}

HL-60 and U937 $\left(1 \times 10^{6}\right.$ cells/dish) were plated in 6-cm dish and then treated with or without CA. After $24 \mathrm{~h}$ incubation, the harvested cells were washed with PBS and fixed with $1 \%$ glutaraldehyde for $30 \mathrm{~min}$. After washing with PBS, the cells were stained with Hoechst 33258 for $10 \mathrm{~min}$. The cells were washed with PBS, and their nuclear morphology was observed by fluorescent microscopy (Eclipse E600, Nikon, Tokyo, Japan).

\subsection{DNA Fragmentation Analysis}

HL-60 and U937 $\left(1 \times 10^{6}\right.$ cells/dish) were plated in 6-cm dish and then treated with or without CA. After the treatments, the cells were washed with ice-cold PBS and resuspended in lysis buffer (50 $\mathrm{mM}$ Tris- $\mathrm{HCl}, \mathrm{pH} 8.0,10 \mathrm{mM}$ EDTA and $0.5 \%$ SDS) with $0.2 \mathrm{mg} / \mathrm{mL}$ RNase A for $30 \mathrm{~min}$ at $50{ }^{\circ} \mathrm{C}$. Proteinase $\mathrm{K}$ was added, and the cells were incubated overnight. The DNA was separated using a $2 \%$ agarose gel and visualized under UV illumination after staining with ethidium bromide. 


\subsection{Flow Cytometry Analysis of Apoptotic Cells}

Cell cycle analysis to detect the sub-G1 phase cells was performed using a cell cycle phase determination kit (Cayman Chemical, Ann Arbor, MI, USA), according to the manufacturer's instructions. HL-60 $\left(1 \times 10^{6}\right.$ cells/dish $)$ was plated in $6-\mathrm{cm}$ dish and then treated with or without CA. After treatment with CA for $12 \mathrm{~h}$ or $24 \mathrm{~h}$, the cells were centrifuged and washed twice with assay buffer. Then, the cells were fixed with fixative and suspended with staining solution containing propidium iodide (PI) and RNase A. The sub-G1 peak was measured and analyzed in the FL2 channel of a FACSCalibur flow cytometer (Becton Dickinson, San Jose, CA, USA) with a $488 \mathrm{~nm}$ excitation laser. The cells $\left(1 \times 10^{4}\right.$ cells $)$ were counted for each sample.

\subsection{Western Blot Analysis}

HL-60 $\left(1 \times 10^{6}\right.$ cells/dish $)$ was plated in 6-cm dish. After treatment with CA for various time periods, the harvested cells were lysed, and the supernatants were boiled for $5 \mathrm{~min}$. The protein concentration was determined using a dye-binding protein assay kit, according to the manufacturer's manual (Bio-Rad, Richmond, CA, USA). Equal amounts of lysate protein were subjected to SDS-PAGE. The proteins were electrotransferred to PVDF membranes and detected, as described previously [32].

\subsection{Preparation of Cytosolic and Mitochondrial Fractions}

HL-60 $\left(1 \times 10^{6}\right.$ cells/dish $)$ were treated with CA for various periods. To prepare the cytosolic fraction, cells were harvested, washed with PBS and resuspended in ice-cold homogenizing buffer (250 mM sucrose, 20 mM HEPES-KOH (pH 7.5), $10 \mathrm{mM} \mathrm{KCl,} 1.5 \mathrm{mM} \mathrm{MgCl} 2,1 \mathrm{mM}$ EDTA, $1 \mathrm{mM}$ EGTA, $1 \mathrm{mM}$ DTT, $1 \mathrm{mM}$ PMSF and protease inhibitors). After $30 \mathrm{~min}$ incubation on ice, the cell lysates were homogenized for 40 strokes, centrifuged at $100,000 \mathrm{~g}$ for $60 \mathrm{~min}$ and the cytosolic fractions were collected. The mitochondrial fraction was isolated using a mitochondria isolation kit, according to the manufacturer's instructions (Thermo Scientific, Rockford, IL, USA). Equal amounts of cytosolic and mitochondrial proteins were used for the Western blotting.

\subsection{Flow Cytometry Analysis of the Mitochondrial Membrane Potential $\left(\Delta \psi_{\mathrm{m}}\right)$}

The mitochondrial membrane potential was analyzed using a JC-1 mitochondrial membrane potential detection kit (Minneapolis, MN, USA). Briefly, the cells $\left(1 \times 10^{6}\right.$ cells/dish) were treated with CA for $12 \mathrm{~h}$ or $24 \mathrm{~h}$, and a JC-1 staining solution was added to culture medium. After 30 min of incubation at $37^{\circ} \mathrm{C}$, the cells were centrifuged and washed twice with assay buffer. The mitochondrial membrane potential was analyzed using a JC-1 mitochondrial membrane potential detection kit (Minneapolis, MN, USA) with a flow cytometer (FACSCalibur), as described by the manufacturer. The cells $\left(1 \times 10^{4}\right.$ cells $)$ were analyzed at the FL1 channel for green JC-1 monomers and the FL2 channel for red JC-1 J-aggregates with a FACSCalibur flow cytometer (Becton Dickinson, San Jose, CA, USA). 


\subsection{Statistical Analysis}

All data were derived from at least three independent experiments. The results were expressed as the mean \pm SD in all conditions. Differences between groups were analyzed using the Student's $t$-test. $p<0.05$ was considered statistically significant.

\section{Conclusions}

We found that CA was the most potent anti-proliferative triterpene derived from $E$. japonica leaves in leukemia cell lines, but not in normal skin fibroblast cell lines. Analysis of the structure-activity relationships of four triterpenes indicated that the $\mathrm{C}_{2}-\mathrm{C}_{3}$ trans-dihydroxyl group and the $\mathrm{C}_{19}-\mathrm{C}_{20}$ trans-dimethyl group are important for suppressing cell proliferation in leukemia cell lines. We showed that CA effectively induced apoptosis in HL-60 cells, which involves the death receptor pathway and the mitochondrial pathway, because CA induced the activation of caspase- 8 and -9 . Our data also demonstrated that CA-induced caspase- 8 activation triggered mitochondrial dysfunction by inducing tBid-mediated Bax activation. These findings suggest that $\mathrm{CA}$ might be a potential candidate for the development of anti-cancer drugs for use in the treatment of leukemia, and it would be interesting to determine whether CA-induced apoptosis has an in vivo role in anti-cancer activity. We previously reported that crocin [33] and naphthoquinone components from Alkanna tinctoria (L.) Tausch [34] exert the anti-cancer effects on human colorectal cancer cells. Further studies are needed to compare the anti-proliferative activity of CA and these compounds.

A number of studies have investigated the anti-cancer potential of triterpenes and their anti-inflammatory, anti-proliferative and pro-apoptotic effects both in vitro and in vivo [35]. Some of the triterpenes strongly induced apoptosis by altering the mitochondria membrane potential and regulating the expression of Bcl-2 family, survivin proteins and modulating the activation of different caspases [35-37]. Since these bioactive triterpenes seem to act on multiple signaling pathways and cell surface receptors, the in vivo and in vitro molecular functional analysis of triterpenes are complicated [35-37]. More recently, it has been reported that UA directly bound to CD36, a class B scavenger receptor, in CHO cells stably transected with CD36 [38] and RAW264.7 macrophages $[39,40]$. Future investigations are needed to compare the binding affinity between CD36 and four triterpenes of E. japonica leaves for deeply understanding the structure-activity correlations.

\section{Acknowledgments}

The research was partly supported by "Science and Technology Research Partnership for Sustainable Development (SATREPS)" of Japan Science and Technology Agency (JST) and the Japan International Cooperation Agency (JICA).

\section{Conflict of Interest}

The authors declare no conflict of interest. 


\section{References}

1. Perry, L.M. Medicinal Plants of East and Southeast Asia; the MIT Press: Cambridge, MA, USA, 1980; pp. 342-343.

2. Uto, T.; Suangkaew, N.; Morinaga, O.; Kariyazono, H.; Oiso, S.; Shoyama, Y. Eriobotryae folium extract suppresses LPS-induced iNOS and COX-2 expression by inhibition of NF- $\kappa \mathrm{B}$ and MAPK activation in murine macrophages. Am. J. Chin. Med. 2010, 38, 985-994.

3. Kang, S.C.; Lee, C.M.; Choi, H.; Lee, J.H.; Oh, J.S.; Kwak, J.H.; Zee, O.P. Evaluation of oriental medicinal herbs for estrogenic and antiproliferative activities. Phytother. Res. 2006, 20, 1017-1019.

4. Kim, M.S.; You, M.K.; Rhuy, D.Y.; Kim, Y.J.; Baek, H.Y.; Kim, H.A. Loquat (Eriobotrya japonica) extracts suppress the adhesion, migration and invasion of human breast cancer cell line. Nutr. Res. Pract. 2009, 3, 259-264.

5. Cha, D.S.; Shin, T.Y.; Eun, J.S.; Kim, D.K.; Jeon, H. Anti-metastatic properties of the leaves of Eriobotrya japonica. Arch. Pharm. Res. 2011, 34, 425-436.

6. Shimizu, M.; Fukumura, H.; Tsuji, H.; Tanaami, S.; Hayashi, T.; Morita, N. Anti-inflammatory constituents of topically applied crude drugs. I. Constituents and anti-inflammatory effect of Eriobotrya japonica LINDL. Chem. Pharm. Bull. 1986, 34, 2614-2617.

7. Ito, H.; Kobayashi, E.; Li, S.H.; Hatano, T.; Sugita, D.; Kubo, N.; Shimura, S.; Itoh, Y.; Tokuda, H.; Nishino, H.; et al. Antitumor activity of compounds isolated from leaves of Eriobotrya japonica. J. Agric. Food Chem. 2002, 50, 2400-2403.

8. Huang, Y.; Li, J.; Cao, Q.; Yu, S.C.; Lv, X.W.; Jin, Y.; Zhang, L.; Zou, Y.H.; Ge, J.F. Anti-oxidative effect of triterpene acids of Eriobotrya japonica (Thunb.) Lindl. leaf in chronic bronchitis rats. Life Sci. 2006, 78, 2749-2757.

9. Lü, H.; Chen, J.; Li, W.L.; Ren, B.R.; Wu, J.L.; Kang, H.Y.; Zhang, H.Q.; Adams, A.; de Kimpe, N. Hypoglycemic and hypolipidemic effects of the total triterpene acid fraction from Folium Eriobotryae. J. Ethnopharmacol. 2009, 122, 486-491.

10. Banno, N.; Akihisa, T.; Tokuda, H.; Yasukawa, K.; Taguchi, Y.; Akazawa, H.; Ukiya, M.; Kimura, Y.; Suzuki, T.; Nishino, H. Anti-inflammatory and antitumor-promoting effects of the triterpene acids from the leaves of Eriobotrya japonica. Biol. Pharm. Bull. 2005, 28, 1995-1999.

11. Lee, C.H.; Wu, S.L.; Chen, J.C.; Li, C.C.; Lo, H.Y.; Cheng, W.Y.; Lin, J.G.; Chang, Y.H.; Hsiang, C.Y.; Ho, T.Y. Eriobotrya japonica leaf and its triterpenes inhibited lipopolysaccharide-induced cytokines and inducible enzyme production via the nuclear factor- $\mathrm{B}$ signaling pathway in lung epithelial cells. Am. J. Chin. Med. 2008, 36, 1185-1198.

12. Ge, J.F.; Wang, T.Y.; Zhao, B.; Lv, X.W.; Jin, Y.; Peng, L.; Yu, S.C.; Li, J. Anti-inflammatory effect of triterpenoic acids of Eriobotrya japonica (Thunb.) Lindl. leaf on rat model of chronic bronchitis. Am. J. Chin. Med. 2009, 37, 309-321.

13. Yang, Y.; Huang, Y.; Huang, C.; Lv, X.; Liu, L.; Wang, Y.; Li, J. Antifibrosis effects of triterpene acids of Eriobotrya japonica (Thunb.) Lindl. leaf in a rat model of bleomycin-induced pulmonary fibrosis. J. Pharm. Pharmacol. 2012, 64, 1751-1760.

14. Fulda, S.; Debatin, K.M. Extrinsic versus intrinsic apoptosis pathways in anticancer chemotherapy. Oncogene 2006, 25, 4798-4811. 
15. Martinou, J.C.; Youle, R.J. Mitochondria in apoptosis: Bcl-2 family members and mitochondrial dynamics. Cell 2011, 21, 92-101.

16. Ho, H.Y.; Lin, W.C.; Kitanaka, S.; Chang, C.T. Analysis of bioactive triterpenes in Eriobotrya japonica lindl. by high-performance liquid chromatography. J. Food Drug Anal. 2008, $16,41-45$.

17. Taniguchi, S.; Imayoshi, Y.; Kobayashi, E.; Takamatsu, Y.; Ito, H.; Hatano, T.; Sakagami, H.; Tokuda, H.; Nishino, H.; Sugita, D.; et al. Production of bioactive triterpenes by Eriobotrya japonica calli. Phytochemistry 2002, 59, 315-323.

18. Lee, M.S.; Cha, E.Y.; Thuong, P.T.; Kim, J.Y.; Ahn, M.S.; Sul, J.Y. Down-regulation of human epidermal growth factor receptor $2 /$ neu oncogene by corosolic acid induces cell cycle arrest and apoptosis in NCI-N87 human gastric cancer cells. Biol. Pharm. Bull. 2010, 33, 931-937.

19. Xu, Y.; Ge, R.; Du, J.; Xin, H.; Yi, T.; Sheng, J.; Wang, Y.; Ling, C. Corosolic acid induces apoptosis through mitochondrial pathway and caspase activation in human cervix adenocarcinoma HeLa cells. Cancer Lett. 2009, 284, 229-237.

20. Fujiwara, Y.; Komohara, Y.; Ikeda, T.; Takeya, M. Corosolic acid inhibits glioblastoma cell proliferation by suppressing the activation of signal transducer and activator of transcription-3 and nuclear factor- $\mathrm{KB}$ in tumor cells and tumor-associated macrophages. Cancer Sci. 2011, 102, 206-211.

21. Wang, J.; Li, Y.; Wang, X.; Jiang, C. Ursolic acid inhibits proliferation and induces apoptosis in human glioblastoma cell lines U251 by suppressing TGF- $\beta 1 /$ miR-21/PDCD4 pathway. Basic Clin. Pharmacol. Toxicol. 2012, 111, 106-112.

22. Reyes-Zurita, F.J.; Rufino-Palomares, E.E.; Lupiáñez, J.A.; Cascante, M. Maslinic acid, a natural triterpene from Olea europaea L., induces apoptosis in HT29 human colon-cancer cells via the mitochondrial apoptotic pathway. Cancer Lett. 2009, 273, 44-54.

23. Juan, M.E.; Planas, J.M.; Ruiz-Gutierrez, V.; Daniel, H.; Wenzel, U. Antiproliferative and apoptosis-inducing effects of maslinic and oleanolic acids, two pentacyclic triterpenes from olives, on HT-29 colon cancer cells. Br. J. Nutr. 2008, 100, 36-43.

24. Tung, N.H.; Shoyama, Y. Eastern blotting analysis and isolation of two new dammarane-type saponins from American ginseng. Chem. Pharm. Bull. 2012, 60, 1329-1333.

25. Shang, M.Y.; Tian, M.; Tanaka, H.; Li, X.W.; Cai, S.Q.; Shoyama, Y. Quality control oftraditional chinese medicine by monoclonal antibody method. Curr. Drug Discov. Technol. 2011, 8, 60-65.

26. Tanaka, H.; Fukuda, N.; Yahara, S.; Isoda, S.; Yuan, C.S.; Shoyama, Y. Isolation of ginsenoside $\mathrm{Rb} 1$ from Kalopanax pictus by eastern blotting using anti-ginsenoside Rb1 monoclonal antibody. Phytother. Res. 2005, 19, 255-258.

27. Tanaka, H.; Fukuda, N.; Shoyama, Y. Eastern blotting and immunoaffinity concentration using monoclonal antibody for ginseng saponins in the field of traditional Chinese medicines. J. Agric. Food Chem. 2007, 55, 3783-3787.

28. Ulukaya, E.; Acilan, C.; Yilmaz, Y. Apoptosis: Why and how does it occur in biology? Cell Biochem. Funct. 2011, 29, 468-480.

29. Armstrong, J.S. Mitochondria: A target for cancer therapy. Br. J. Pharmacol. 2006, 147, 239-248.

30. Von Ahsen, O.; Waterhouse, N.J.; Kuwana, T.; Newmeyer, D.D.; Green, D.R. The "harmless" release of cytochrome c. Cell Death Differ. 2000, 7, 1192-1199. 
31. Crompton, M. Bax, Bid and the permeabilization of the mitochondrial outer membrane in apoptosis. Curr. Opin. Cell Biol. 2000, 12, 414-419.

32. Uto, T.; Morinaga, O.; Tanaka, H.; Shoyama, Y. Analysis of the synergistic effect of glycyrrhizin and other constituents in licorice extract on lipopolysaccharide-induced nitric oxide production using knock-out extract. Biochem. Biophys. Res. Commun. 2012, 417, 473-478.

33. Aung, H.H.; Wang, C.Z.; Ni, M.; Fishbein, A.; Mehendale, S.R.; Xie, J.T.; Shoyama, Y.; Yuan, C.S. Crocin from Crocus sativus possesses significant anti-proliferation effects on human colorectal cancer cells. Exp. Oncol. 2007, 29, 175-180.

34. Tung, N.H.; Du, G.J.; Wang, C.Z.; Yuan, C.S.; Shoyama, Y. Naphthoquinone components from Alkanna tinctoria (L.) Tausch show significant antiproliferative effects on human colorectal cancer cells. Phytother. Res. 2013, 27, 66-70.

35. Patlolla, J.M.; Rao, C.V. Triterpenoids for cancer prevention and treatment: Current status and future prospects. Curr. Pharm. Biotechnol. 2012, 13, 147-155.

36. Bishayee, A.; Ahmed, S.; Brankov, N.; Perloff, M. Triterpenoids as potential agents for the chemoprevention and therapy of breast cancer. Front Biosci. 2011, 16, 980-996.

37. Fulda, S. Modulation of apoptosis by natural products for cancer therapy. Planta Med. 2010, 76, 1075-1079.

38. Wilkinson, K.; Boyd, J.D.; Glicksman, M.; Moore, K.J.; El Khoury, J. A high content drug screen identifies ursolic acid as an inhibitor of amyloid beta protein interactions with its receptor CD36. J. Biol. Chem. 2011, 286, 34914-34922.

39. Murakami, A.; Ohnishi, K. Target molecules of food phytochemicals: Food science bound for the next dimension. Food Funct. 2012, 3, 462-476.

40. Ikeda, Y.; Murakami, A.; Fujimura, Y.; Tachibana, H.; Yamada, K.; Masuda, D.; Hirano, K.; Yamashita, S.; Ohigashi, H. Aggregated ursolic acid, a natural triterpenoid, induces IL-1beta release from murine peritoneal macrophages: Role of CD36. J. Immunol. 2007, 178, 4854-4864.

(C) 2013 by the authors; licensee MDPI, Basel, Switzerland. This article is an open access article distributed under the terms and conditions of the Creative Commons Attribution license (http://creativecommons.org/licenses/by/3.0/). 